\title{
Dietary intake and cardiometabolic risk factors among Venezuelan adults: a nationally representative analysis
}

Dina Goodman', Juan P. González-Rivas 1,2,3, Lindsay M. Jaacks', Maritza Duran³, María Inés Marulanda 3,4, Eunice Ugel ${ }^{3,5}$, Josiemer Mattei ${ }^{6}$, Jorge E. Chavarro ${ }^{6}$ and Ramfis Nieto-Martinez ${ }^{1,3,7^{*}}$

\begin{abstract}
Background: Increasing trends in global obesity have been attributed to a nutrition transition where healthy foods are replaced by ultra-processed foods. It remains unknown if this nutrition transition has occurred in Venezuela, a country undergoing a socio-political crisis with widespread food shortages.

Methods: We described dietary intake of Venezuelans from a nationally representative study conducted between 2014 and 2017. We conducted a cross-sectional analysis of dietary, sociodemographic, and clinical data from Venezuelans $\geq 20$ years of age $(n=3420)$. Dietary intake was assessed using a semi-quantitative food frequency questionnaire. Standardized clinical and anthropometric measurements estimated obesity, type 2 diabetes, and hypertension. A Dietary Diversity Score (DDS) was calculated using an amended Minimum Dietary Diversity for Women score where the range was 0 to 8 food groups, with 8 being the most diverse. Analyses accounted for complex survey design by estimating weighted frequencies of dietary intake and DDS across sociodemographic and cardiometabolic risk-based subgroups.

Results: The prevalence of obesity was $24.6 \%$ (95\% Cl: 21.6-27.7), type 2 diabetes was 13.3\% (11.2-15.7), and hypertension was 30.8\% (27.7-34.0). Western foods were consumed infrequently. Most frequently consumed foods included coffee, arepas (a salted corn flour cake), and cheese. Mean DDS was 2.3 food groups (Range: 0-8, Standard Error: 0.07) and this score did not vary among subgroups. Men, younger individuals, and those with higher socioeconomic status were more likely to consume red meat and soft drinks once or more weekly. Women and those with higher socioeconomic status were more likely to consume vegetables and cheese once or more daily. Participants with obesity, type 2 diabetes, and hypertension had lower daily intake of red meat and arepas compared to participants without these risk factors.

(Continued on next page)
\end{abstract}

\footnotetext{
* Correspondence: nietoramfis@hsph.harvard.edu

'Department of Global Health and Population, Harvard TH Chan School of Public Health, Boston, MA, USA

${ }^{3}$ Foundation for Clinic, Public Health, and Epidemiology Research of Venezuela (FISPEVEN INC), Caracas, Venezuela

Full list of author information is available at the end of the article
}

(c) The Author(s). 2020 Open Access This article is licensed under a Creative Commons Attribution 4.0 International License, which permits use, sharing, adaptation, distribution and reproduction in any medium or format, as long as you give appropriate credit to the original author(s) and the source, provide a link to the Creative Commons licence, and indicate if changes were made. The images or other third party material in this article are included in the article's Creative Commons licence, unless indicated otherwise in a credit line to the material. If material is not included in the article's Creative Commons licence and your intended use is not permitted by statutory regulation or exceeds the permitted use, you will need to obtain permission directly from the copyright holder. To view a copy of this licence, visit http://creativecommons.org/licenses/by/4.0/. The Creative Commons Public Domain Dedication waiver (http://creativecommons.org/publicdomain/zero/1.0/) applies to the data made available in this article, unless otherwise stated in a credit line to the data. 
(Continued from previous page)

Conclusions: Despite high prevalence of cardiometabolic risk factors, adults in Venezuela have not gone through a nutrition transition similar to that observed elsewhere in Latin America. Dietary diversity is low and widely consumed food groups that are considered unhealthy are part of the traditional diet. Future studies are needed in Venezuela using more comprehensive measurements of dietary intake to understand the effect of the sociopolitical crisis on dietary patterns and cardiometabolic risk factors.

Keywords: Nutrition transition, Western diet, Obesity, Venezuela, Latin America

\section{Background}

The relationship between suboptimal dietary intake and noncommunicable diseases (NCDs) is well-established. In 2017, 11 million deaths and 255 million disability-adjusted life years (DALYs) were attributable to dietary risk factors, a considerable increase from an estimated 8 million deaths and 184 DALYs in 1990 [1]. Concurrently, the global prevalence of obesity, as well as related cardiometabolic diseases such as type 2 diabetes (T2D) and hypertension, increased substantially over the past 40 years [2].

The nutrition transition [3, 4] describes the process where a high prevalence of undernutrition is replaced by overnutrition through large changes in dietary intake and physical activity patterns, resulting in a diet primarily consisting of westernized, ultra-processed foods $[5,6]$. Though long believed to only affect individuals with high socioeconomic status, the obesity epidemic has increasingly spread to lower socioeconomic groups [7]. Several Latin American countries, including Argentina [7], Mexico [8, 9], and Brazil [10, 11] have demonstrated this shifting burden across socioeconomic groups [7].

Venezuela is a particularly salient case study as it is currently undergoing an economic and socio-political crisis that has led to widespread food shortages and malnutrition [12], factors that may reverse the nutrition transition. Prior to the crisis, the burdens of T2D, hypertension, and obesity were documented to be increasing over time, particularly in urban areas [13, 14]. However, few studies have looked at recent dietary intake in Venezuela $[15,16]$ and most of these have been limited to convenience samples rather than nationally representative data. Furthermore, NCD burden has been especially hard to quantify over the past few years, as the Venezuelan government has stopped publishing national statistics since 2016 [17].

This paper aims to describe the relationship between dietary intake and obesity, hypertension, and T2D, using a nationally representative dataset from EVESCAM (Estudio Venezolano de Salud Cardio-Metabólica). EVES CAM, conducted between 2014 and 2017, was the first nationally-representative study in Venezuela on risk factors for cardiometabolic disease, with data on NCDs including T2D, obesity, and hypertension; and lifestyle risk factors for these diseases [18].

\section{Methods}

\section{Study population}

Data are from EVESCAM, a population-based, clustersampled study. Details of the study design and sampling strategy have been published elsewhere [18]. Briefly, between July 2014 and January 2017, 4454 study participants were enrolled through a multi-stage stratified sampling method, using parish as the primary sampling unit. As such, these data are representative at the national level. Enrollment occurred at the household-level, where all members aged $\geq 20$ years were invited to participate. Exclusion criteria included pregnancy, inability to stand or communicate, or refusal to participate.

\section{Dietary intake assessment}

Dietary intake was ascertained using a semi-quantitative food frequency questionnaire that was developed through a working group of Venezuelan nutrition experts and hosted by the EVESCAM principal investigators. The questionnaire (Additional file 1) asked participants to list frequency of consumption and portion size for 33 food groups, based on show cards used to help estimate portion sizes accurately (Additional file 2). Responses were categorized by frequency: daily ( 1 portion, $2-4$ portions, or $\geq 5$ portions per day), weekly (1 portion, 2-4 portions, or $\geq 5$ portions per week), or monthly (0 portion, 1-3 portions). Responses were converted to frequency of daily intake using the median value of each category (e.g. 2-4 portions per week was recoded as 3 times per week or 0.43 times per day; 1-3 times per month was recoded as 2 times per month or 0.07 times per day). Water, sugar, and alcohol were excluded from this analysis.

A Dietary Diversity Score (DDS) was calculated to indicate the number of different food categories that participants reported consuming. This score was calculated based on Minimum Dietary Diversity-Women (MDD-W) developed by the Food and Agriculture Organization of the United Nations [19]. Based on the food groups collected in EVESCAM, the DDS used eight food categories rather than ten in MDDW. This analysis categorized food groups as: 1) grains, white roots and tubers, plantains; 2) pulses; 3) Nuts and seeds; 4) Dairy; 5) Meat-based foods: red 
meat, poultry and fish; 6) Eggs; 7) Fruits 8) Vegetables. Food groups included in each category are listed in Table 4 (Appendix 1). Each food category was given a score of zero if consumed weekly or less or one if at least one portion was consumed daily, and then a final score was created by summing all eight categories. Thus, a score of eight represents the most diverse diet and zero the least diverse diet.

\section{Covariate assessment}

Sociodemographic variables included sex, age, and socioeconomic status (SES). SES was calculated using a version of the Graffar Scale modified for Venezuela [20], which pools income, profession, educational level, and housing conditions into a composite score. Each variable is rated independently from one to five, with one being the highest level of SES. A final score sums the independent ratings and classifies participants' SES as high, medium-high, medium, medium-low, and low [20]. Few participants were in the highest quintile $(1.3 \%)$ so the two highest categories were merged. Data on sex, age, and SES were missing for $<5 \%$ of participants.

Weight was measured with the lightest possible clothes, without shoes, using a calibrated scale (Tanita UM-081 $1^{\circ}$, Japan). Height was measured using a portable stadiometer (Seca $206^{\circ}$ Seca GmbH \& Co., Hamburg, Germany). Body mass index (BMI) was defined as weight (measured in kilograms) divided by height (measured in meters) squared, and classified as underweight $(<18.5$ $\left.\mathrm{kg} / \mathrm{m}^{2}\right)$, normal weight $\left(18.5\right.$ to $\left.<25.0 \mathrm{~kg} / \mathrm{m}^{2}\right)$, overweight $\left(25.0\right.$ to $\left.<30.0 \mathrm{~kg} / \mathrm{m}^{2}\right)$, or obesity $\left(\geq 30.0 \mathrm{~kg} / \mathrm{m}^{2}\right)[21]$.

Blood pressure was measured twice, in five-minute intervals, in the right arm using a validated oscillometric sphygmomanometer (Omron HEM-705C Pint $^{\circ}$ Omron Healthcare CO., Kyoto/Japan) [22]. Participants were seated and rested their arm at heart level. Hypertension was defined as having a systolic blood pressure $\geq 140$ $\mathrm{mmHg}$, diastolic blood pressure $\geq 90 \mathrm{mmHg}$, or selfreport of antihypertensive medication use [23].

Blood glucose measurements included fasting blood glucose and a 2-h oral glucose tolerance test (OGTT) using a 300-ml test solution containing $75 \mathrm{~g}$ anhydrous glucose. Diabetes was defined as either: fasting plasma glucose $\geq 126 \mathrm{mg} / \mathrm{dL}, 2$-h after $75 \mathrm{~g}$ oral glucose tolerance test $\geq 200 \mathrm{mg} / \mathrm{dL}$, or self-report of diabetes medication use [24]. The mean age of diagnosis was 50.6 years and $7 \%$ of participants were on insulin only, so we refer to all participants as having T2D. Sensitivity analyses excluding one participant diagnosed at $<21$ years of age and on insulin only did not change results (data not shown).

\section{Statistical analysis}

Of 4454 participants screened, 3420 were enrolled in EVESCAM and included in the present analysis. As food group consumption was not always normally distributed, reporting only mean daily consumption would have been statistically inaccurate. For this reason, medians and interquartile ranges (IQR) were graphically represented using boxplots, one set of boxplots showing median consumption of Western foods and another for traditional foods. Western foods (eight total) included white bread, red meat, cookies, cake, soft drinks, fast food, french fries, and burgers [4]. Traditional foods (17 total) were determined based on previous studies categorizing Venezuelan dietary patterns [13], and included arepas (a salted corn flour cake), coffee, cheese, white rice, vegetables, fruits, fruit juice, empanadas, oats, legumes, fish, poultry, eggs, plantains, potatoes, pasta, and soup. Cereal, cachapa, and nuts were not included in the boxplots as they were consumed monthly or less by over $75 \%$ of participants.

Bivariate analyses accounting for complex survey design were conducted to estimate dietary intakes by sociodemographic characteristics, including age, sex, and SES, and clinical subgroups (i.e. body mass, hypertension, and T2D status) using Pearson chi-squared tests. Differences between mean DDS by subgroup were evaluated using Somers' D, a rank-sum test appropriate for weighted data [25]. This test also calculates jackknife standard error, adjusted for the primary sampling unit. Food groups were included in the bivariate analysis if they had a skewed distribution (Figs. 1 and 2) to understand if their consumption might differ by subgroups and if they were consumed weekly or daily by $>25 \%$ of participants.

All analyses were performed in Stata 16.0 (College Station, Texas, USA).

\section{Results}

Weighted characteristics of all participants, and by sex, are summarized in Table 1 . Briefly, participants $(n=$ 3420 ) were between 20 and 96 years of age, with a mean age of 41.2 years (Standard Deviation (SD): 0.67) and $52.3 \%$ were female. The majority of participants lived in urban areas; only 19.3\% of participants lived in rural areas. Most participants had BMI classified as overweight (34.5\%; 95\% Confidence Interval (CI): 31.8-37.4) or normal weight $(36.6 \%$; $32.5-40.7)$. Weighted prevalence of obesity was $24.6 \%$ (95\% CI: 21.6-27.7), T2D $13.3 \%$ (11.2-15.7), and hypertension 30.8\% (27.7-34.0). Underweight (4.3\%; 95\% CI: 3.2-5.8), extreme poverty (5.6\%; 3.8-8.3), and high SES (2.0\%; 0.9-4.4) were uncommon in the sample. Compared to females, there were significantly more male participants with T2D (15.9\% of males vs $10.9 \%$ of females, $p$-value $<0.001)$ and hypertension (32.8\% of males vs $28.9 \%$ of females, pvalue $=0.055)$. 


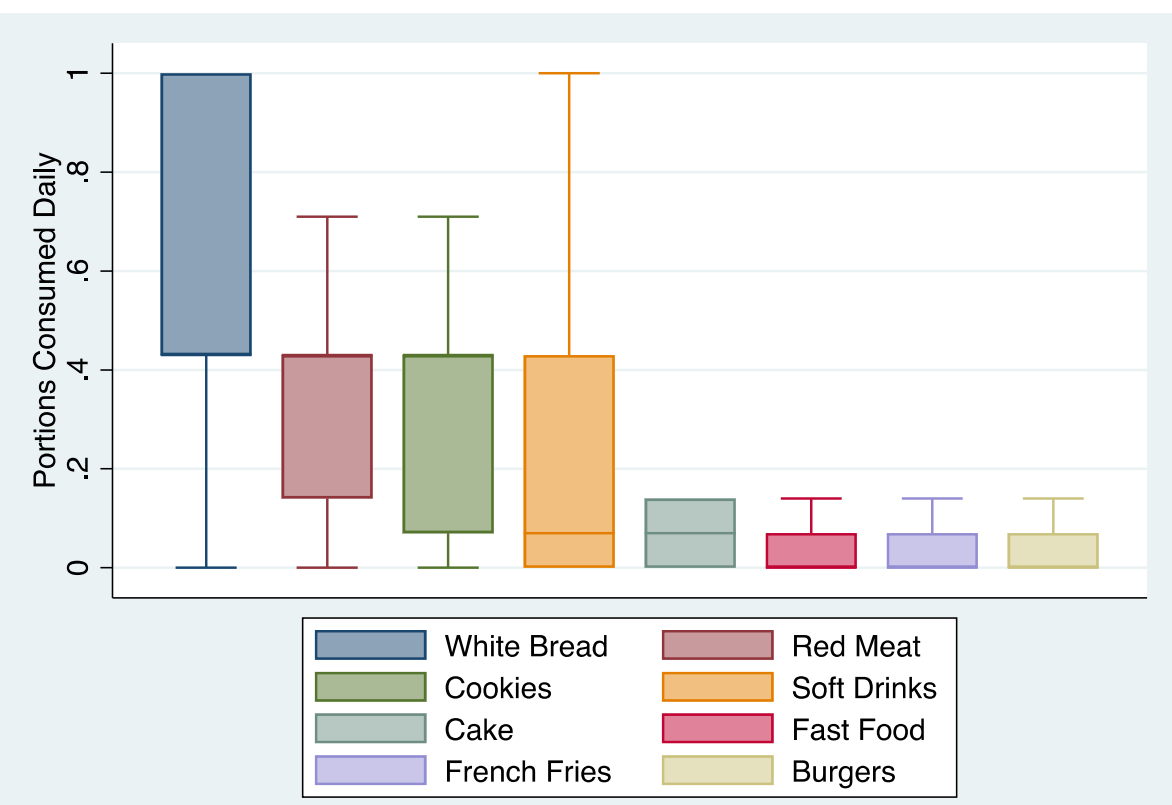

Fig. 1 Boxplot of consumption of Western foods among Venezuelan adults, 2014-2017. The values displayed here are median and interquartile range. This figure excludes outliers. Food groups are displayed as portions consumed daily

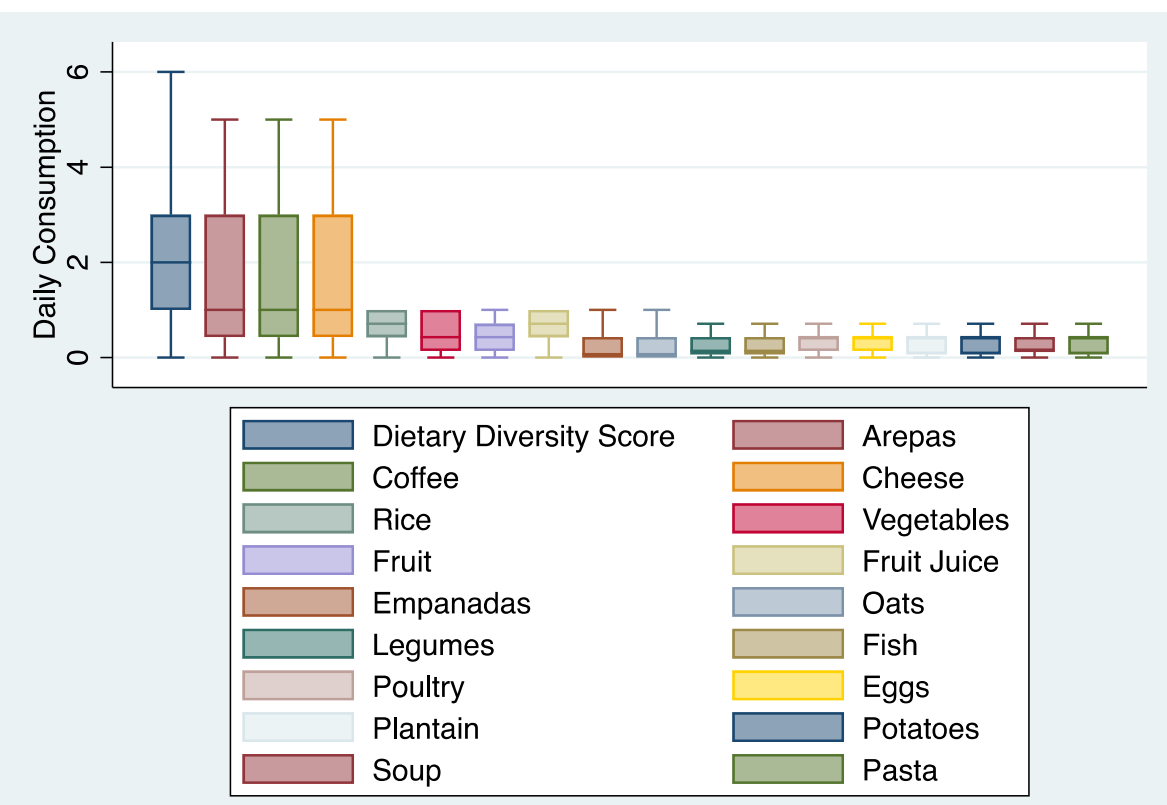

Fig. 2 Boxplots of consumption of traditional food groups and distribution of Dietary Diversity Score among Venezuelan adults, 2014-2017. The values displayed here are medians and interquartile ranges. This figure excludes outliers. Dietary diversity score was calculated as an amended minimum dietary diversity-women (MDD-W) score, where each food category was given a score of 0 if consumed weekly or less or 1 if at least one portion was consumed daily, and then a final score was created by summing all eight categories. Food groups included in each category are listed in Appendix 1 in Table 4. Individual food groups are displayed as portions consumed daily 
Table 1 Sociodemographic and clinical characteristics of adults*

\begin{tabular}{|c|c|c|c|c|}
\hline & \multicolumn{3}{|c|}{ Weighted \% (95\%Cl) } & \multirow{2}{*}{$\begin{array}{l}P \text { - } \\
\text { value }^{1}\end{array}$} \\
\hline & Total $\left(n^{2}=3402\right)$ & Male $\left(n^{2}=1052\right)$ & Female $\left(n^{2}=2348\right)$ & \\
\hline Overall & & $47.7(45.2-50.3)$ & $52.3(49.7-54.8)$ & \\
\hline Socioeconomic Status ${ }^{3}$ & & & & 0.320 \\
\hline High \& Medium-High & $21.2(17.1-25.9)$ & $22.3(17.3-28.3)$ & $20.1(16.4-24.5)$ & \\
\hline Medium & $31.0(27.8-34.3)$ & $30.7(26.6-35.2)$ & $31.2(28.1-34.5)$ & \\
\hline Relative Poverty & $42.2(37.4-47.1)$ & $40.7(35-46.7)$ & $43.6(38.8-48.5)$ & \\
\hline Extreme poverty & $5.6(3.8-8.4)$ & $5.1(3.4-7.5)$ & $5.1(3.4-7.5)$ & \\
\hline Age Category & & & & 0.034 \\
\hline $20-34$ & $40.3(36.0-44.6)$ & $38.7(32.9-44.9)$ & $41.7(37.3-46.3)$ & \\
\hline $35-44$ & $21.5(19.1-24.1)$ & $20.9(17.3-25.1)$ & $22.1(20.1-24.2)$ & \\
\hline $45-54$ & $17.0(15.6-18.6)$ & $17.4(15.1-19.9)$ & $16.7(15.1-18.5)$ & \\
\hline $55-64$ & $11.4(10.1-12.8)$ & $11.1(9.6-12.9)$ & $11.6(10.1-13.4)$ & \\
\hline$\geq 65$ & $9.8(8.0-11.8)$ & $11.8(9.7-14.4)$ & $7.9(6.3-9.8)$ & \\
\hline Locality & & & & 0.047 \\
\hline Rural & $19.3(9.1-36.4)$ & $17.7(8.4-33.6)$ & $20.8(9.7-39.0)$ & \\
\hline Urban & 80.7 (63.6-90.9) & $82.3(66.4-91.6)$ & $79.2(61.0-90.3)$ & \\
\hline BMI Category ${ }^{4}$ & & & & 0.008 \\
\hline Underweight & $4.3(3.2-5.8)$ & $3.5(2.3-5.3)$ & $5.1(3.6-7.2)$ & \\
\hline Normal Weight & $36.6(32.5-40.7)$ & $36.2(30.3-42.4)$ & $36.9(33.7-40.2)$ & \\
\hline Overweight & $34.5(31.8-37.4)$ & $38.2(34.3-42.2)$ & $31.2(28.5-34.2)$ & \\
\hline Obesity & $24.6(21.7-27.7)$ & $22.2(18.2-26.8)$ & $26.7(24.2-29.4)$ & \\
\hline Type 2 Diabetes 5 & $13.3(11.2-15.7)$ & $15.9(13.2-19.0)$ & $10.9(8.9-13.3)$ & $<0.001$ \\
\hline Hypertension $^{6}$ & $30.8(27.7-34.0)$ & $32.8(29.2-37.2)$ & $28.9(25.7-32.2)$ & 0.055 \\
\hline
\end{tabular}

"Estimates are weighted to be representative of Venezuelan adults over 20 years of age

$1 P$-values calculated using Pearson's chi-square

$2 \mathrm{n}=$ unweighted sample size

${ }^{3}$ SES was calculated using a version of the Graffar Scale modified for Venezuela [19], which combines income, profession, educational level, and housing conditions into a composite score

${ }^{4}$ BMI was defined as weight (measured in kilograms) divided by height (measured in meters) squared, and classified as underweight $\left(<18.5 \mathrm{~kg} / \mathrm{m}^{2}\right)$, normal weight $\left(18.5\right.$ to $\left.<25.0 \mathrm{~kg} / \mathrm{m}^{2}\right)$, overweight $\left(25.0\right.$ to $\left.<30.0 \mathrm{~kg} / \mathrm{m}^{2}\right)$, or obesity $\left(\geq 30.0 \mathrm{~kg} / \mathrm{m}^{2}\right)[21]$

${ }^{5}$ Type 2 diabetes was defined by either: fasting plasma glucose was $\geq 126 \mathrm{mg} / \mathrm{dL}$, 2-h after $75 \mathrm{~g}$ oral glucose tolerance test $\geq 200 \mathrm{mg} / \mathrm{dL}$, or self-report of diabetes medications [24]

${ }^{6}$ Hypertension was defined as having a systolic blood pressure $\geq 140 \mathrm{mmHg}$, diastolic blood pressure $\geq 90 \mathrm{mmHg}$, or self-report of antihypertensive medication use [23]

\section{Consumption of Western \& Traditional Foods}

White bread had the most frequent and variable consumption of the Western food groups (Fig. 1), with a median consumption of approximately three portions weekly (Median: 0.43, IQR: 0.43-1 portions daily). Red meat and cookies also had median consumption of three portions weekly but lower IQRs than white bread (red meat: 0.140.43 portions daily; cookies: $0.07-0.43$ portions daily). Soft drinks had variable consumption patterns, despite low median consumption of 0.07 portions daily or approximately two portions monthly. Consumption of cake, french fries, burgers, and fast food was infrequent, with over $75 \%$ participants reporting consuming these food groups monthly or less.

Across all participants, median DDS was 2.00 (IQR: 13 ). The most commonly consumed traditional foods were arepas, coffee, and cheese, each with a median consumption of one time daily (IQR: $0.43-3$ portions daily) (Fig. 2).

\section{Distribution of consumption by sociodemographic subgroups}

Weighted bivariate analyses were conducted for DDS, white bread, red meat, cookies, soft drinks, arepas, coffee, cheese, vegetables, and fruits (Tables 2 and 3). As shown in Table 2, mean DDS was 2.28 (SE: 0.07) and did not vary by any sociodemographic subgroups.

Daily consumption patterns for a number of food groups differed by sex, namely white bread, cookies, soft drinks, cheese, and vegetables. Compared to females, a higher proportion of males reporting daily consumption of white bread (35\% of males vs $29 \%$ of females; $p=$ 


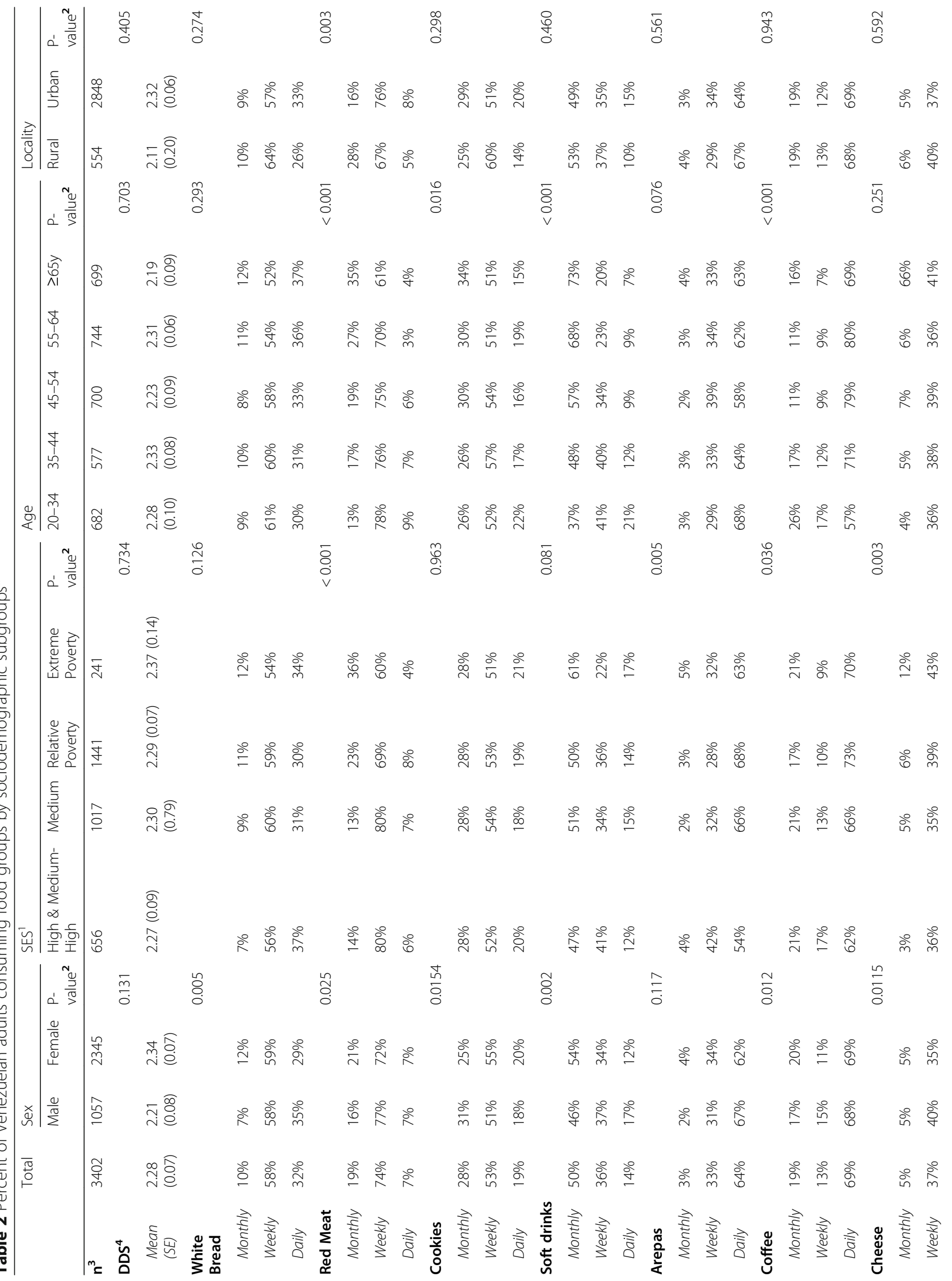




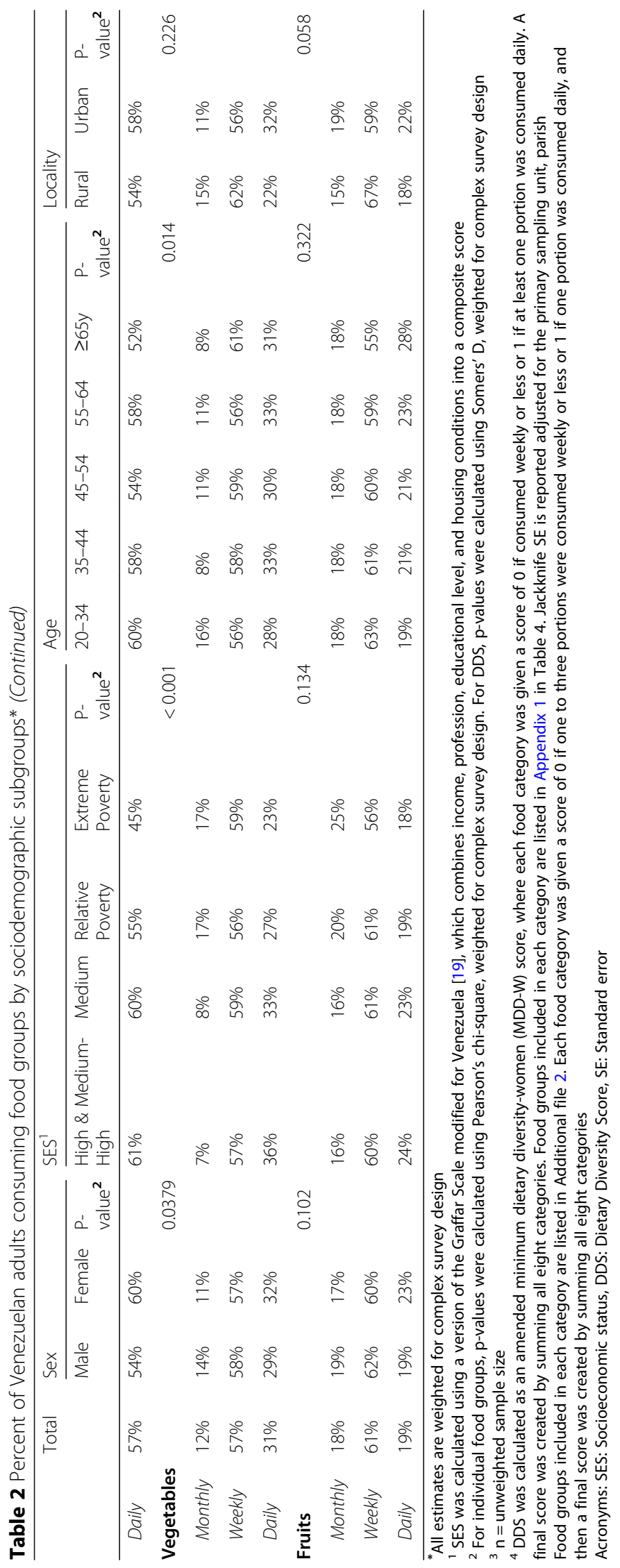


Table 3 Percent of Venezuelan adults consuming food groups by cardiometabolic risk status*

\begin{tabular}{|c|c|c|c|c|c|c|c|c|c|c|c|c|}
\hline & Total & BMI Category ${ }^{1}$ & & & & & Type 2 Diak & etes $^{2}$ & & Hypertensic & & \\
\hline & & Under-weight & Normal weight & Overweight & Obesity & P-value ${ }^{4}$ & No & Yes & P-value ${ }^{4}$ & No & Yes & $P$-value ${ }^{4}$ \\
\hline$\overline{n^{5}}$ & 3402 & 133 & 1198 & 1204 & 853 & & 2790 & 601 & & 1908 & 1493 & \\
\hline $\mathrm{DDS}^{5}$ & & & & & & 0.315 & & & 0.630 & & & 0.879 \\
\hline Mean (SE) & $2.28(0.07)$ & $2.62(0.17)$ & $2.31(0.08)$ & $2.18(0.08)$ & $2.29(0.08)$ & & $2.29(0.06)$ & $2.21(0.14)$ & & $2.27(0.07)$ & $2.29(0.08)$ & \\
\hline White Bread & & & & & & 0.405 & & & 0.456 & & & 0.605 \\
\hline Monthly & $10 \%$ & $8 \%$ & $10 \%$ & $10 \%$ & $9 \%$ & & $10 \%$ & $10 \%$ & & $10 \%$ & $10 \%$ & \\
\hline Weekly & $58 \%$ & $57 \%$ & $58 \%$ & $61 \%$ & $57 \%$ & & $59 \%$ & $56 \%$ & & $59 \%$ & $57 \%$ & \\
\hline Daily & $32 \%$ & $36 \%$ & $33 \%$ & $29 \%$ & $35 \%$ & & $32 \%$ & $35 \%$ & & $31 \%$ & $34 \%$ & \\
\hline Red Meat & & & & & & 0.033 & & & 0.006 & & & 0.002 \\
\hline Monthly & $19 \%$ & $32 \%$ & $18 \%$ & $19 \%$ & $17 \%$ & & $18 \%$ & $24 \%$ & & $17 \%$ & $22 \%$ & \\
\hline Weekly & $74 \%$ & $58 \%$ & $76 \%$ & $75 \%$ & $75 \%$ & & $75 \%$ & $72 \%$ & & $76 \%$ & $70 \%$ & \\
\hline Daily & $7 \%$ & $10 \%$ & $7 \%$ & $6 \%$ & $8 \%$ & & $7 \%$ & $4 \%$ & & $7 \%$ & $7 \%$ & \\
\hline Cookies & & & & & & 0.481 & & & 0.848 & & & 0.214 \\
\hline Monthly & $28 \%$ & $18 \%$ & $28 \%$ & $28 \%$ & $30 \%$ & & $28 \%$ & $29 \%$ & & $27 \%$ & $30 \%$ & \\
\hline Weekly & $53 \%$ & $57 \%$ & $54 \%$ & $54 \%$ & $50 \%$ & & $53 \%$ & $52 \%$ & & $53 \%$ & $53 \%$ & \\
\hline Daily & $19 \%$ & $25 \%$ & $19 \%$ & $18 \%$ & $20 \%$ & & $19 \%$ & $18 \%$ & & $20 \%$ & $17 \%$ & \\
\hline Soft drinks & & & & & & 0.2916 & & & $<0.001$ & & & $<0.001$ \\
\hline Monthly & $50 \%$ & $51 \%$ & $53 \%$ & $50 \%$ & $46 \%$ & & $48 \%$ & $64 \%$ & & $46 \%$ & $59 \%$ & \\
\hline Weekly & $36 \%$ & $29 \%$ & $35 \%$ & $37 \%$ & $35 \%$ & & $38 \%$ & $24 \%$ & & $38 \%$ & $29 \%$ & \\
\hline Daily & $14 \%$ & $19 \%$ & $13 \%$ & $14 \%$ & $14 \%$ & & $15 \%$ & $13 \%$ & & $16 \%$ & $11 \%$ & \\
\hline Arepas & & & & & & 0.013 & & & 0.025 & & & 0.043 \\
\hline Monthly & $3 \%$ & $1 \%$ & $4 \%$ & $2 \%$ & $3 \%$ & & $3 \%$ & $2 \%$ & & $3 \%$ & $3 \%$ & \\
\hline Weekly & $33 \%$ & $25 \%$ & $30 \%$ & $33 \%$ & $38 \%$ & & $32 \%$ & $39 \%$ & & $31 \%$ & $36 \%$ & \\
\hline Daily & $64 \%$ & $74 \%$ & $66 \%$ & $65 \%$ & $59 \%$ & & $65 \%$ & $60 \%$ & & $66 \%$ & $61 \%$ & \\
\hline Coffee & & & & & & 0.789 & & & 0.009 & & & 0.075 \\
\hline Monthly & $19 \%$ & $18 \%$ & $20 \%$ & $17 \%$ & $19 \%$ & & $20 \%$ & $14 \%$ & & $20 \%$ & $17 \%$ & \\
\hline Weekly & $13 \%$ & $15 \%$ & $13 \%$ & $12 \%$ & $12 \%$ & & $13 \%$ & $8 \%$ & & $14 \%$ & $10 \%$ & \\
\hline Daily & $69 \%$ & $67 \%$ & $67 \%$ & $70 \%$ & $69 \%$ & & $67 \%$ & $78 \%$ & & $67 \%$ & $73 \%$ & \\
\hline Cheese & & & & & & 0.451 & & & 0.0929 & & & 0.875 \\
\hline Monthly & $5 \%$ & $5 \%$ & $6 \%$ & $5 \%$ & $4 \%$ & & $5 \%$ & $4 \%$ & & $14 \%$ & $11 \%$ & \\
\hline Weekly & $37 \%$ & $37 \%$ & $40 \%$ & $37 \%$ & $35 \%$ & & $37 \%$ & $42 \%$ & & $58 \%$ & $57 \%$ & \\
\hline Daily & $57 \%$ & $58 \%$ & $55 \%$ & $58 \%$ & $61 \%$ & & $58 \%$ & $54 \%$ & & $29 \%$ & $32 \%$ & \\
\hline Vegetables & & & & & & 0.011 & & & 0.478 & & & 0.5011 \\
\hline Monthly & $12 \%$ & $19 \%$ & $14 \%$ & $12 \%$ & $7 \%$ & & $12 \%$ & $11 \%$ & & $13 \%$ & $11 \%$ & \\
\hline Weekly & $58 \%$ & $59 \%$ & $55 \%$ & $58 \%$ & $60 \%$ & & $58 \%$ & $56 \%$ & & $57 \%$ & $58 \%$ & \\
\hline Daily & $30 \%$ & $22 \%$ & $31 \%$ & $30 \%$ & $33 \%$ & & $30 \%$ & $33 \%$ & & $30 \%$ & $31 \%$ & \\
\hline Fruits & & & & & & 0.099 & & & 0.8128 & & & 0.4633 \\
\hline Monthly & $18 \%$ & $15 \%$ & $19 \%$ & $17 \%$ & $19 \%$ & & $18 \%$ & $17 \%$ & & $17 \%$ & $20 \%$ & \\
\hline Weekly & $61 \%$ & $54 \%$ & $58 \%$ & $64 \%$ & $61 \%$ & & $60 \%$ & $62 \%$ & & $61 \%$ & $59 \%$ & \\
\hline Daily & $21 \%$ & $31 \%$ & $24 \%$ & $18 \%$ & $20 \%$ & & $21 \%$ & $21 \%$ & & $22 \%$ & $21 \%$ & \\
\hline
\end{tabular}

*All estimates are weighted for complex survey design

${ }^{1}$ BMl category was defined as weight (measured in kilograms) divided by height (measured in meters) squared, and classified as underweight $\left(<18.5 \mathrm{~kg} / \mathrm{m}^{2}\right)$, normal weight $\left(18.5\right.$ to $\left.<25.0 \mathrm{~kg} / \mathrm{m}^{2}\right)$, overweight $\left(25.0\right.$ to $\left.<30.0 \mathrm{~kg} / \mathrm{m}^{2}\right)$, or obesity $\left(\geq 30.0 \mathrm{~kg} / \mathrm{m}^{2}\right)[21]$

${ }^{2}$ Type 2 diabetes was defined by either: fasting plasma glucose was $\geq 126 \mathrm{mg} / \mathrm{dL}, 2$-h after $75 \mathrm{~g}$ oral glucose tolerance test $\geq 200 \mathrm{mg} / \mathrm{dL}$, or self-report of diabetes [24] ${ }^{3}$ Hypertension was defined as having a systolic blood pressure $\geq 140 \mathrm{mmHg}$, diastolic blood pressure $\geq 90 \mathrm{mmHg}$, or self-report of antihypertensive medication use [23] ${ }^{4}$ For individual food groups, $p$-values were calculated using Pearson's chi-square, weighted for complex survey design. For DDS, $p$-values were calculated using Somers' D, weighted for complex survey design

$5 \mathrm{n}=$ unweighted sample size

${ }^{6}$ DDS was calculated as an amended minimum dietary diversity-women (MDD-W) score, where each food category was given a score of 0 if consumed weekly or less or 1 if at least one portion was consumed daily. A final score was created by summing all eight categories. Food groups included in each category are listed in Appendix 1 in Table 4. Jackknife SE is reported adjusted for the primary sampling unit, parish

Acronyms: BMI: body mass index, DDS: Dietary Diversity Score, SE: Standard error 
$0.005)$ and soft drinks (17\% vs $12 \% p=0.002)$. However, females consumed more cookies (18\% of males vs $20 \%$ of females; $p=0.0154)$, cheese ( $54 \%$ vs $60 \% ; p=0.0115)$, and vegetables $(29 \%$ vs $32 \% ; p=0.0379)$ daily. There was no difference by sex for consumption patterns of fruit $(p=0.102)$ or arepas $(p=0.117)$.

Consumption patterns of white bread $(p=0.126)$, cookies $(p=0.963)$, soft drinks $(p=0.081)$, and fruits (0.134) did not vary by SES. Participants with higher SES consumed more red meat weekly compared to those with lower SES $(p<0.001)$ : weekly consumption was $80 \%$ among those in the high \& medium-high and medium categories, $69 \%$ among those in relative poverty, and $60 \%$ among those in extreme poverty. A similar pattern was observed for daily consumption of cheese ( $p=$ $0.003)$ and vegetables $(p<0.0001)$ where daily consumption was highest among those with high \& medium-high SES (61\% for cheese and 36\% for vegetables in the highest SES category) compared to consumption among those in lower SES categories (45\% for cheese and 23\% for vegetables in the lowest SES category). However, consumption patterns were less linear for daily consumption of coffee and arepas. Daily arepa consumption was highest among participants in relative poverty (68\%) and medium SES (66\%), followed by participants in extreme poverty (63\%), compared to participants in the high \& medium-high category $(54 \%)(p=0.005)$. Generally, lower SES category correlated with higher daily intake of coffee $(p=0.036)$ : daily coffee consumption was $73 \%$ among those in relative poverty and $70 \%$ among those in extreme poverty, compared to $66 \%$ among those in the medium SES category and $62 \%$ in the high and medium-high category.

Consumption patterns of white bread $(p=0.293)$, cheese $(p=0.251)$, and fruits $(p=0.322)$ did not vary by age category. However, certain food groups were consumed more frequently by younger age groups compared to others. For instance, younger participants consumed more red meat weekly compared to older participants $(p<0.001)$ : weekly consumption was $78 \%$ among those $<35$-years-old and $75 \%$ among $45-54$-year-olds, compared to $61 \%$ among > 65-year-olds. Similarly, younger participants consumed more soft drinks weekly than older participants $(\mathrm{p}<$ 0.001 ): weekly consumption was $41 \%$ among those $<35$ years-old, compared to $23 \%$ among 55-64-year-olds and $20 \%$ among $>65$-year-olds. Weekly vegetable consumption, however, was higher among older age categories $(p=$ 0.014 ) with $61 \%$ of those $>65$ years, $56 \%$ of those $55-65$ years, and $59 \%$ of those $45-54$ years consuming vegetables weekly, compared to $58 \%$ of those $35-44$ and $56 \%<35$ years of age.

The majority of food group consumption did not vary significantly by locality, with the exception of red meat $(p=0.003)$. Red meat was most likely to be consumed weekly among those living in urban areas (76\%) compared to those in rural areas (67\%). Fruit consumption bordered statistical significance $(p=0.058)$ and at least one portion of fruit was more likely to be consumed weekly in rural areas (67\%) compared to urban areas (59\%).

\section{Distribution of consumption by cardiometabolic risk status}

DDS did not differ by cardiometabolic risk status. Frequency of food group intake did differ by BMI category for red meat $(p=0.033)$, arepas $(p=0.013)$, and vegetables $(p=0.011)$. Daily consumption of red meat and arepas generally decreased with increasing BMI category. For red meat, $10 \%$ of participants with an underweight BMI reported daily consumption and $7 \%$ of participants with normal weight, compared to $6 \%$ of participants with an overweight BMI. Participants with obesity, however, had slightly higher red meat consumption with $8 \%$ of participants reporting daily consumption. Daily arepa consumption decreased with increasing BMI category, with $74 \%$ of participants with an underweight BMI consuming arepas daily, compared to $66 \%$ with a normal BMI, $65 \%$ with an overweight BMI, and 59\% with obesity. One-third of participants with obesity had daily vegetable intake compared to $30 \%$ with an overweight BMI and $22 \%$ of participants with an underweight BMI. There was no difference in vegetable intake by hypertension $(p=0.57)$ or T2D status $(p=0.68)$.

Consumption of red meat, soft drinks, arepas, and coffee differed significantly by T2D status. Those with T2D were less likely to consume red meat, soft drinks, and arepas daily: $4 \%$ of participants with T2D consumed red meat weekly compared to $7 \%$ without T2D $(p=0.006)$, $13 \%$ of those with T2D consumed soft drinks daily compared to $15 \%$ without $(p<0.001)$, and $60 \%$ of those with T2D consumed arepas daily compared $65 \%$ of those without. Daily coffee consumption, however, was more frequent among participants with T2D (78\%) compared to those without T2D (67\%).

Similarly, consumption of red meat, soft drinks, and arepas differed significantly by hypertension status. While daily consumption of red meat was $7 \%$ for both those participants with hypertension and those without, weekly consumption was $76 \%$ among those without hypertension compared to $70 \%$ among those with hypertension $(p=0.002)$. Daily consumption of soft drinks $(\mathrm{p}<0.001)$ and arepas $(0.043)$ were both higher among participants without hypertension: $16 \%$ of those without hypertension consumed soft drinks daily compared to $11 \%$ of those with hypertension, and $66 \%$ of those without hypertension consumed arepas daily compared to $61 \%$ of those with hypertension. 


\section{Discussion}

This nationally representative descriptive analysis of dietary patterns of Venezuelans in 2014-2017 found that the study population had a high prevalence of obesity, T2D, and hypertension. Most frequently consumed food groups included white bread, arepas, coffee, and cheese. Intake of many Western foods were relatively low, with over $75 \%$ of participants consuming french fries, burgers, and fast foods only monthly or less frequently.

In general, this analysis found females had healthier diets than males, with lower consumption of white bread, red meat, and soft drinks, although dietary diversity was similar between the sexes. There were inconsistent differences in intake by SES category: those with higher SES, compared to those in relative or extreme poverty, consumed more portions of some healthy foods (e.g. higher daily intake of fruit and vegetables), but also unhealthy foods (e.g. higher daily intake of white bread and cheese). Overall, younger and urban Venezuelans ate more Western foods than both older and rural Venezuelans.

Overall, dietary diversity was very low. Gomez et al. (2019), a study of eight Latin American countries, found that other countries have much more diverse diets with DDS scores of five to six (out of nine) [15]. They found a slightly higher mean score in Venezuela than this analysis (5.62 of 9 compared to 2.3 of 8 ), but their study was conducted in only urban areas and during an earlier time period (2014-2015) than EVESCAM (2014-2017) which may have been affected by the onset of the humanitarian crisis in Venezuela.

Healthier food groups did not differ substantially by BMI category or T2D or hypertension status, while unhealthier foods (namely red meat, cheese, and arepas) were consumed more frequently by participants classified with overweight BMI and obesity, but less frequently by individuals with T2D and hypertension. This may suggest that people with diagnosed T2D and hypertension may follow positive behaviour change and nutritional recommendations made upon diagnosis [26]. Furthermore, individuals with obesity consumed more vegetables daily and consumed fewer soft drinks and cookies, potentially reflecting attempts to lose weight.

Although consumption of Western foods was low, the most commonly consumed food groups are not considered 'healthy' by most dietary guidelines for the region and worldwide [27]. White bread, arepas, and cheese dominated daily consumption in this nationally representative sample. In general, the composition of the cheeses in Venezuela have high fat content [13] and may be a factor contributing to obesity and T2D. In fact, a recent study in the US found that increasing cheese consumption by $>0.5$ servings per day was associated with 9\% (95\% CI: 2-16) higher risk of T2D, compared to maintaining usual cheese consumption [28].
These results suggest that the nutrition transition has not influenced the dietary intake of Venezuela as markedly as other Latin American countries, such as Brazil and Mexico [5, 8]. This could be due to the economic crisis in Venezuela, which started in 2014 and led to government restrictions on foreign products [29]. Meanwhile, traditional foods have long been subsidized by the government, possibly increasing their availability and consumption. Arepas, in particular, are considered a staple food in Venezuela. They are prepared from a cornmeal that is fortified with vitamin A and iron. The glycemic index is relatively high (74), though lower than white wheat bread (98) [30], however, to our knowledge, no studies have specifically evaluated the prospective association between arepas intake and obesity or T2D. However, given strong evidence linking refined grains to these outcomes [31], one might posit that arepas may also increase risk and substituting for whole grains may decrease risk. Unfortunately, it is unlikely that such substitutions would be culturally acceptable.

Despite markedly low consumption of Western, ultraprocessed foods, the prevalence of obesity (24.6\%) was higher in this sample than the regional average: in 2014, $19 \%$ of adults in Latin America were classified with obesity [32]. However, adult obesity prevalence remains lower in Venezuela than in Mexico or Chile, both with an obesity prevalence of approximately 35\% in 2014 [33]. Furthermore, obesity prevalence found in this sample is lower than previous estimates from before the socio-political crisis. For example, the weighted prevalence of obesity was $27.9 \%$, when calculated from all population-based studies published between 1999 and 2010 which included a total of 8584 individuals [13]. In a previous regional analysis that included the EVESCAM cohort but did not include any dietary data, obesity prevalence in Venezuela did not vary by urban versus rural areas and was generally highest in middle quartiles of educational attainment and lowest in the bottom quartile [7].

In the present study, obesity prevalence was higher among women than men (26.7\% versus $22.2 \%)$, a trend that matches obesity patterns worldwide and in other parts of Latin America [5, 7]. Although some aspects of the diet in this Venezuelan cohort were healthier among women, the higher prevalence of obesity could be explained by several factors including body size preferences for women in Latino cultures [34], lower physical activity levels (particularly work-related physical activity) [35], parity and resultant excess gestational weight gain/ post-partum weight retention, and potentially genetic and/or hormonal differences [7].

While prevalence for hypertension in our study was comparable to previous studies, the estimates for diabetes were higher. Here, the weighted prevalence was 
13.1\% (95\% CI: $11.2-15.7)$, for T2D and 30.8\% (27.734.0) for hypertension. Other studies reported diabetes prevalence to be about $8 \%$ and hypertension to be about $30 \%[36,37]$. Differences in definitions of T2D and sampling may explain some of these differences, however this warrants further study using longitudinal data.

This study has a number of strengths. First, EVES CAM is the first nationally representative study of Venezuelan diet and provides a better understanding of the nuances in dietary patterns throughout Latin America, particularly in a country undergoing a socio-political crisis and that has been neglected in global nutrition literature. This analysis provides nationally-representative estimates of diabetes, obesity, and hypertension prevalence during a time period where health data has been sparse in Venezuela. Further, a number of diverse traditional food groups specific to the study context, such as arepas, empanadas, and fried bananas, were included in the questionnaire, as well as pictures of portion sizes to ensure that participants could more accurately selfreport the frequency and number of portions consumed.

Nonetheless, study design limitations must be taken into consideration. First, we cannot definitively rule out type 1 diabetes cases but globally over 95\% of diabetes cases are T2D [38]. In this sample, however, very few participants were on insulin (7\%) and average age of diagnosis was 50.6 years, so this was unlikely to be a major source of error in this analysis. Second, this crosssectional study relied on self-reporting of semiquantitative nutritional data, which is prone to recall bias and impedes the ability to make causal inferences from this analysis. The semi-quantitative nature of the questionnaire also limited our ability to calculate caloric intake from each food group. In general, countries around the world are increasingly shifting towards foodbased dietary guidelines [39]. It was outside the aims and scope of this study to explore nutrient data such as energy and so the instrument used was not suitable to capture nutrient data. Finally, the questionnaire used organized answer options in a manner that may have increased measurement error. Specifically, frequency of consumption was in increasing order within daily, weekly, and monthly categories. However, this questionnaire was completed with the guidance of strictly trained enumerators who supervised that answer choices matched the participants' consumption patterns using showcards with portion sizes.

Further studies that employ validated quantitative methods to measure diet may assist in assessing diet more accurately, as well as conducting nutrient analysis. Moreover, future studies could also include recipes and preparation styles to better disaggregate ingredients included in mixed dishes. Lastly, since this study is conducted in a vulnerable population undergoing a

\section{Appendix 1}

Table 4 Food Categories Used for Dietary Diversity Score

\begin{tabular}{ll}
\hline Food Category for MDD-W & Food Group in EVESCAM DDS \\
\hline $\begin{array}{l}\text { Grains, white roots and } \\
\text { tubes, plantains }\end{array}$ & $\begin{array}{l}\text { White bread, pasta, cereal, oats, rice, } \\
\text { potato, plantain, yucca } \\
\text { Pulses }\end{array}$ \\
$\begin{array}{l}\text { Luts \& Seeds } \\
\text { Nuts }\end{array}$ \\
$\begin{array}{l}\text { Dairy products } \\
\text { Animal-based foods }\end{array}$ & Filk, cheese \\
Eggs & Eggs \\
Other Fruits & Fruit \\
Vitamin-A rich fruits & \\
Other Vegetables & Vegetables \\
Leafy green vegetables & \\
\hline
\end{tabular}

humanitarian crisis, longitudinal data is needed to understand how food patterns have changed since baseline data collection in 2014-2017, when the crisis was already ongoing but less extensive. Diets might have changed among individuals with new cases of T2D or hypertension or with exacerbated complications of these conditions. Furthermore, though obesity was clearly a national problem in Venezuela at the time of data collection, this may have changed with food insecurity.

\section{Conclusions}

In summary, this nationally representative, cross-sectional analysis suggests that that dietary intake Venezuela are quite different from other countries in Latin America which have a high reliance on soft drinks and ultraprocessed foods. Nutrition policy measures throughout Latin America should be tailored to consumption patterns and socio-political contexts of each country.

\section{Supplementary information}

Supplementary information accompanies this paper at https://doi.org/10. 1186/s40795-020-00362-7.

Additional file 1. Dietary Intake Questionnaire.

Additional file 2. Show Cards Used in the EVESCAM Study.

\section{Abbreviations}

BMI: Body mass index; Cl: Confidence Interval; DALYs: Disability-adjusted life years; DDS: Dietary Diversity Score; EVESCAM: Estudio Venezolano de Salud Cardio-Metabólica; IQR: Interquartile ranges; MDD-W: Minimum Dietary Diversity-Women; NCDs: Non-communicable diseases; OGT: Oral glucose tolerance test; SES: Socioeconomic status

\section{Acknowledgements}

Not applicable.

Authors' contributions

DG wrote the first draft, performed the statistical analyses, and produced tables and figures. RNM is the PI of EVESCAM and conceived the idea of the study. 
JPGR, JM, LMJ, and JC provided guidance for the analysis and contributed to writing the final manuscript. JPGR, MD, MIM, and EU participated in dataset preparation, assisted with data collection and reviewed the results of this analysis. All authors read and approved the final manuscript.

\section{Funding}

The EVESCAM study was partially funded by a grant of Novartis Venezuelan and private donations. This analysis was not funded.

\section{Availability of data and materials}

The datasets used and/or analysed during the current study are available from the corresponding author on reasonable request.

\section{Ethics approval and consent to participate}

The study protocol complied with the Helsinki declaration and was approved by the National Bioethics Committee (CENABI) of Venezuela and this secondary analysis was approved by the Harvard T.H. Chan School of Public Health (protocol \#: IRB19-1538). Written informed consent was obtained from all participants. The present report is presented according to the Strengthening the Reporting of Observational Studies in Epidemiology (STROBE) [40].

\section{Consent for publication}

There is no identifiable information of the study participants in the present manuscript, therefore it is not applicable.

\section{Competing interests}

The authors declare that they have no competing interests.

\section{Author details}

'Department of Global Health and Population, Harvard TH Chan School of Public Health, Boston, MA, USA. ${ }^{2}$ International Clinical Research Center (ICRC), St. Ann's University Hospital, Brno, Czech Republic. ${ }^{3}$ Foundation for Clinic, Public Health, and Epidemiology Research of Venezuela (FISPEVEN INC), Caracas, Venezuela. ${ }^{4}$ Endocrine Associates of Florida, Research Department, Orlando, Florida, USA. ${ }^{5}$ Public Health Research Unit, Department of Social and Preventive Medicine, School of Medicine, Universidad Centro-Occidental "Lisandro Alvarado", Barquisimeto, Venezuela. 'Department of Nutrition, Harvard TH Chan School of Public Health, Boston, MA 02115, USA. 'LifeDoc Health, Memphis, TN, USA.

\section{Received: 22 March 2020 Accepted: 15 July 2020}

Published online: 16 October 2020

\section{References}

1. Afshin A, Sur PJ, Fay KA, Cornaby L, Ferrara G, Salama JS, et al. Health effects of dietary risks in 195 countries, 1990-2017: a systematic analysis for the global burden of disease study 2017. Lancet, 2019;393(10184):1958-72.

2. Collaborators GRF. Global, regional, and national comparative risk assessment of 84 behavioural, environmental and occupational, and metabolic risks or clusters of risks for 195 countries and territories, 19902017: a systematic analysis for the Global Burden of Disease Study 2017. Lancet. 2018;392(10159):1923-94.

3. Popkin BM. The nutrition transition in low-income countries: an emerging crisis. Nutr Rev. 1994;52(9):285-98.

4. Popkin BM. Nutritional patterns and transitions. Popul Dev Rev. 1993;19(1): 138-57

5. Jaacks LM, Vandevijvere S, Pan A, McGowan CJ, Wallace C, Imamura F, et al. The obesity transition: stages of the global epidemic. Lancet Diabetes Endocrinol. 2019;7(3):231-40.

6. Popkin BM, Corvalan C, Grummer-Strawn LM. Dynamics of the double burden of malnutrition and the changing nutrition reality. Lancet. 2020; 395(10217):65-74

7. Jiwani SS, Carrillo-Larco RM, Hernández-Vásquez A, Barrientos-Gutiérrez T, Basto-Abreu A, Gutierrez L, et al. The shift of obesity burden by socioeconomic status between 1998 and 2017 in Latin America and the Caribbean: a cross-sectional series study. Lancet Glob Health. 2019;7(12): e1644-e54.

8. Rivera JA, Barquera S, Gonzalez-Cossio T, Olaiz G, Sepulveda J. Nutrition transition in Mexico and in other Latin American countries. Nutr Rev. 2004 62(7 Pt 2):S149-57.
9. Rivera JA, Barquera S, Campirano F, Campos I, Safdie M, Tovar V. Epidemiological and nutritional transition in Mexico: rapid increase of noncommunicable chronic diseases and obesity. Public Health Nutr. 2002;5(1a): 113-22.

10. Conde $\mathrm{WL}$, Monteiro CA. Nutrition transition and double burden of undernutrition and excess of weight in Brazil. Am J Clin Nutr. 2014;100(6): 1617s-22s.

11. Monteiro CA, Mondini $L$, de Souza AL, Popkin BM. The nutrition transition in Brazil. Eur J Clin Nutr. 1995;49(2):105-13.

12. Doocy S, Ververs M-T, Spiegel P, Beyrer C. The food security and nutrition crisis in Venezuela. Soc Sci Med. 2019;226:63-8.

13. Nieto-Martínez R, Hamdy O, Marante D, Inés Marulanda M, Marchetti A, Hegazi RA, et al. Transcultural diabetes nutrition algorithm (tDNA): Venezuelan application. Nutrients. 2014;6(4):1333-63.

14. Nieto-Martínez R, González-Rivas JP, Lima-Martínez M, Stepenka V, Rísquez A, Mechanick J. Diabetes Care in Venezuela. Ann Glob Health. 2015;81(6): 776-91.

15. Gómez G, Fisberg RM, Nogueira Previdelli Á, et al. Diet Quality and Diet Diversity in Eight Latin American Countries: Results from the Latin American Study of Nutrition and Health (ELANS). Nutrients. 2019;11(7):1605.

16. Ekmeiro JS, Moreno RR, García ML, Cámara FM. Food consumption pattern at a family level of urban areas of Anzoátegui. Venezuela. Nutr Hosp. 2015; 32(4):1758-65.

17. Page KR, Doocy S, Reyna Ganteaume F, Castro JS, Spiegel P, Beyrer C. Venezuela's public health crisis: a regional emergency. Lancet. 2019; 393(10177):1254-60.

18. Nieto-Martínez R, Inés Marulanda M, González-Rivas JP, Ugel E, Durán M, Barengo N, et al. Cardio-metabolic health Venezuelan study (EVESCAM): design and implementation. Investigacion Clinica. 2017;58(1):56-69.

19. FAO F. Minimum dietary diversity for women: a guide for measurement. Rome: FAO; 2016.

20. Méndez-Castellano H, Méndez MC. Estratificación social y humana. Método de Graffar modificado. Arch Venez Puer Pediatr. 1986;49:93-104.

21. Clinical Guidelines on the Identification, Evaluation, and Treatment of Overweight and Obesity in Adults--The Evidence Report. National Institutes of Health. Obes Res 1998;6 Suppl 2:51S-209S.

22. Vera-Cala LM, Orostegui M, Valencia-Angel LI, Lopez N, Bautista LE. Accuracy of the Omron HEM-705 CP for blood pressure measurement in large epidemiologic studies. Arq Bras Cardiol. 2011;96(5):393-8.

23. Chobanian AV, Bakris GL, Black HR, Cushman WC, Green LA, Izzo Jr JL, et al. Seventh report of the joint national committee on prevention, detection, evaluation, and treatment of high blood pressure. hypertension. 2003;42(6): 1206-52.

24. American Diabetes Association. 2. Classification and Diagnosis of Diabetes: Standards of Medical Care in Diabetes-2019. Diabetes Care. 2019; 42(Supplement 1):S13.

25. Newson R. Confidence intervals for rank statistics: Somers' D and extensions. Stata J. 2006;6(3):309-34.

26. Mainous AG, Diaz VA, Geesey ME. Acculturation and healthy lifestyle among Latinos with diabetes. Ann Fam Med. 2008;6(2):131-7.

27. FAO. Latin America and the Caribbean. Food and Agriculture Organization of the United Nations.

28. Drouin-Chartier J-P, Li Y, Ardisson Korat AV, Ding M, Lamarche B, Manson JE, et al. Changes in dairy product consumption and risk of type 2 diabetes: results from 3 large prospective cohorts of US men and women. Am J Clin Nutr. 2019;110(5):1201-12.

29. Fraser B, Willer H. Venezuela: aid needed to ease health crisis. Lancet. 2016; 388(10048):947-9.

30. Izquierdo M, Oráa E, de los Ríos V, Drayer R, Urbina D. Índice glucémico e insulinémico de alimentos ricos en carbohidratos. Rev Venezol Endocrinol Metab. 2006:4:25.

31. Reynolds A, Mann J, Cummings J, Winter N, Mete E, Te Morenga L. Carbohydrate quality and human health: a series of systematic reviews and meta-analyses. Lancet. 2019;393(10170):434-45.

32. Collaboration NRF. Trends in adult body-mass index in 200 countries from 1975 to 2014: a pooled analysis of 1698 population-based measurement studies with 19. 2 million participants. Lancet. 2016; 387(10026):1377-96.

33. Kain J, Hernández Cordero S, Pineda D, de Moraes AF, Antiporta D, Collese $\mathrm{T}$, et al. Obesity prevention in Latin America. Curr Obes Rep. 2014;3(2):150-5. 
34. Goodman JR. Flabless is fabulous: how Latina and Anglo women read and incorporate the excessively thin body ideal into everyday experience. J Mass Commun Q. 2002;79(3):712-27.

35. Mattei J, Tamez M, Ríos-Bedoya CF, Xiao RS, Tucker KL, Rodríguez-Orengo JF. Health conditions and lifestyle risk factors of adults living in Puerto Rico: a cross-sectional study. BMC Public Health. 2018;18(1):491.

36. Omboni S, Aristizabal D, De la Sierra A, Dolan E, Head G, Kahan T, et al. Hypertension types defined by clinic and ambulatory blood pressure in 14 143 patients referred to hypertension clinics worldwide. Data from the ARTEMIS study. J Hypertens. 2016;34(11):2187-98.

37. Nieto-Martínez R, González-Rivas JP, Ugel E. Prevalence of cardiometabolic risk factors in three populations from Venezuela: the VEMSOLS STUDY 20062010. Medicas UIS. 2018;31(1):15-22.

38. International Diabetes Federation. IDF Diabetes Atlas 2019. 9th ed; 2019.

39. Herforth A, Arimond M, Alvarez-Sanchez C, Coates J, Christianson K, Muehlhoff E. A global review of food-based dietary guidelines. Adv Nutr. 2019;10(4):590-605.

40. von Elm E, Altman DG, Egger M, Pocock SJ, Gøtzsche PC, Vandenbroucke $J P$, et al. Strengthening the Reporting of Observational Studies in Epidemiology (STROBE) statement: guidelines for reporting observational studies. BMJ (Clinical research ed). 2007;335(7624):806-8.

\section{Publisher's Note}

Springer Nature remains neutral with regard to jurisdictional claims in published maps and institutional affiliations.

Ready to submit your research? Choose BMC and benefit from:

- fast, convenient online submission

- thorough peer review by experienced researchers in your field

- rapid publication on acceptance

- support for research data, including large and complex data types

- gold Open Access which fosters wider collaboration and increased citations

- maximum visibility for your research: over $100 \mathrm{M}$ website views per year

At $\mathrm{BMC}$, research is always in progress.

Learn more biomedcentral.com/submissions 